DOI: $10.21767 / 2573-4482.100017$

\title{
Diffuse Drug-Induced Dermatitis following Sclerotherapy for Telangiectasias
}

\section{Libby MW, Caitlin WH, Deniz OA and Heller JA*}

Department of Surgery, Johns Hopkins Vein Center, Johns Hopkins Medical Centers, Baltimore, USA

*Corresponding author: Jennifer A Heller, 10755 Falls Road, Pavilion 1 Suite 360, Lutherville, MD 21903, Tel: 4105500415; Email: jheller6@jhmi.edu

Received date: June 14, 2016; Accepted date: July 26, 2016; Published date: August 02, 2016

Copyright: (c) 2016 Libby MW, et al. This is an open-access article distributed under the terms of the Creative Commons Attribution License, which permits unrestricted use, distribution, and reproduction in any medium, provided the original author and source are credited.

\section{Abstract}

Sclerotherapy is an effective treatment modality for the management of lower extremity telangiectasias. Although localized dermatologic reactions are known complications of this procedure, systemic reactions are rare. Here, we present a case of a diffuse drug eruption following sclerotherapy for the treatment of bilateral lower extremity telangiectasias. Salient clinical and physical exam findings are described. Management strategies for the treatment of drug eruptions are outlined. This is the first case report that we know of describing a diffuse drug eruption associated with sclerotherapy. It is important to recognize the possibility of diffuse drug eruptions secondary to sclerotherapy treatment so that expectant management may be initiated expeditiously in affected patients.

\section{Case Report}

A 64-year-old woman with hypertension and bilateral lower extremity telangiectasias presented from an outside facility following several sclerotherapy injections using polidocanol over a three-month period. Following the first session, she developed a diffuse eczematous rash over the bilateral lower extremities associated with pruritis and erythema (Figure 1). She had no history of dermatitis prior to this episode. As sclerotherapy sessions continued, the rash progressed to involve the torso, neck, and upper extremities, sparing her face and dorsum of her hands. She denied any recent travel, change in detergent, soaps, or perfumes.

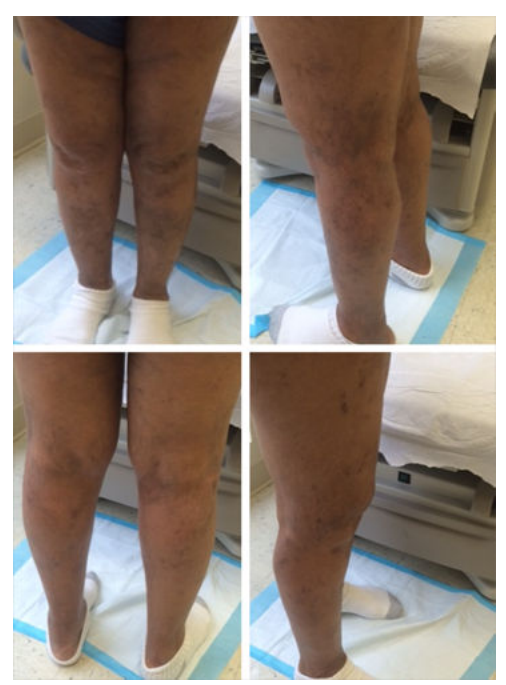

Figure 1: Drug eruption following sclerotherapy.

The patient was referred for evaluation by a dermatologist, who initially diagnosed diffuse dermatitis based on clinical exam. She was prescribed a series of treatment modalities including antibiotics, antihistamines, and steroids without improvement. Subsequent biopsies of the rash (Figure 2) revealed hyperkeratosis with foci of parakeratosis, acanthosis, spongiosis, focal basilar vacuolar alteration, and scattered apoptotic keratinocytes throughout the specimen. There were many pseudo-pautrier microabcesses containing histiocytes and Langerhans cells, as well as foci of lymphocyte exocytosis, some associated with spongiosis and alignment with the basilar epidermis. The dermis contained a mild perivascular lymphohistiocytic infiltrate and scattered pigmented macrophages. Taken together, these findings were consistent with a lymphocytic drug reaction with mild lymphocytic atypia. 


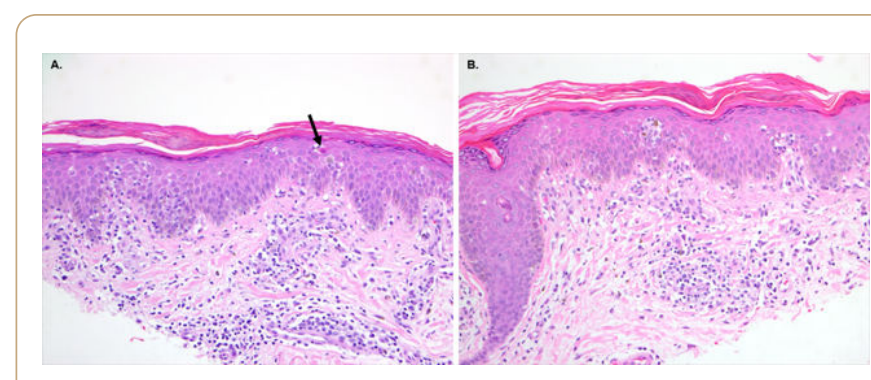

Figure 2: Pathology slides demonstrating lymphocytic drug reaction following sclerotherapy injection for telangiectasias. Panel A: Parakeratosis, hyperkeratosis, lymphocyte extravasation with spongiosis, and focal basilar vacuolar alteration in epidermis. On right upper epidermis an apoptotic keratinocyte is seen (arrow). Panel B:

Parakeratosis hyperkeratosis and pseudo-pautrier microabscess containing histiocytic clusters in epidermis. In both panels, the dermis is noted to have a mild to moderate perivascular lyphohistiocytic infiltrate and pigmented macrophages. Slides are presented at 200x magnification.

A thorough review of the patient's history revealed that her anti-hypertensive regimen had recently changed from lisinopril to amlodipine just prior to her first sclerotherapy session. Although the etiology of the drug eruption was unclear, the patient was transitioned back to lisinopril and started on triamcinolone ointment and hydroxyzine for symptomatic relief. She experienced near-complete resolution of her druginduced dermatitis within 2 weeks of these changes.

Several weeks later, the patient was restarted on amlodipine in an effort to optimize her blood pressure control. Her dermatitis did not worsen with the reintroduction of amlodipine. She has had no further sclerotherapy sessions, and remains dermatitis-free at this time. Based on these findings, it appears that injection of sclerosant may have been responsible for the patient's drug eruption.

\section{Discussion}

Dermatologic reactions are rare, but recognized, side effects of many drugs [1-7]. These reactions can be toxic, metabolic, or allergic in nature. Certain patient populations are at increased risk for developing drug eruptions, including women, patients who are immune compromised, and those with autoimmune deficiencies. The most common drug reaction is an exanthematous rash, which accounts for $40 \%$ of all drug eruptions and is often associated with pruritis and fever [8] More serious reactions, such as Stevens-Johnson syndrome, are also known to occur [1]. However, diffuse dermatologic reactions are not a previously recognized side effect of sclerosant injection.

Sclerotherapy is a widely accepted technique to treat both aesthetic and pathophysiologic disorders of the superficial venous system. It is particularly useful for small caliber veins, as the effectiveness of sclerosant is dependent upon the area of the internal lumen of the vein that comes in contact with the injected agent. Studies suggest there may be more than
$70 \%$ improvement in telangiectasias after only two treatment sessions [9]. Sclerotherapy is considered the preferred method of treatment for telangiectasias because it has equivalent or better outcomes than laser therapy, but is better tolerated due to less discomfort $[10,11]$. There are several sclerosing agents available for use, including osmotic, alcohol, and detergent agents. Studies suggest that osmotic and detergent agents have equivalent efficacy in the treatment of telangiectasias, while alcohol agents may have somewhat better efficacy. Polidocanol, the commonly used detergent agent, is known to be associated with less pain than other sclerosing agents [12-14].

Dermatologic eruptions localized to sclerotherapy injection sites have been previously documented. For example, granuloma annulare following sclerotherapy has been described, presumably as the result of trauma to the skin from the injection itself combined with the introduction of foreign material (i.e., sclerosant) [15]. Urticaria at the injection site and telangiectatic matting anywhere on the lower extremity are common side effects related to the injection of sclerosant [16]. Hyperpigmentation can also occur, usually in a dosedependent manner that is directly proportional to the concentration of the sclerosant used.

More severe reactions, such as Nicolau's livedoid dermatitis, infrequently occur following scleroterapy, but are usually confined to the injection site $[17,18]$. Patients affected by this syndrome experience rapid onset of severe pain with erythema, blanching, and a reticular rash at the site of injection, followed skin and deep tissue necrosis that leads to disfiguring scars. The rash does not usually spread systemically, and is much more severe than the patient's reaction that we describe. Systemic allergic reactions can occur with any of the sclerosing agents, but generally present with traditional allergy symptoms such as shortness of breath, edema, and multisystem organ dysfunction [16]. Diffuse drug-induced dermatitis distant from the local area of the injection site is not, to our knowledge, a previously recognized side effect of sclerotherapy.

The most important step in treating a drug eruption is identification and cessation of the offending agent. Additionally, topical corticosteroids and oral antihistamines can be used for symptomatic relief of pruritus or pain. NSAIDs may also be administered to those patients who develop a fever as a component of their reaction [19]. Ultimately, once the offending agent is withdrawn, supportive care is the first line of therapy. Most drug eruptions are self-limited, and will resolve over 2-6 weeks.

\section{Conclusion}

Sclerotherapy is recognized as a safe and effective treatment for lower extremity telangiectasias. Although dermatologic reactions local to the injection site are not uncommon, these are usually mild and self-limited. Rarely, patients may experience more diffuse dermatologic reactions following injection of sclerosant. If unexpected or atypical skin reactions occur, sclerotherapy should be discontinued until an 
underlying etiology has been determined. These patients should be managed expectantly, and treated for symptomatic control.

\section{References}

1. Tuchinda $P$ (2013) Cutaneous adverse reactions to calcium channel blockers. Asian Pac J Allergy Immunol.

2. Baetz BE, Patton ML, Guilday (2011) Amlodipine-Induced toxic Epidermal Necrolysis. J Burn Care Res 32: e158-e160.

3. Grabczynska SA, Cowley N (2000) Amlodipine inducedphotosensitivity presenting as telangiectasia. Br J Dermatol 142: 1255-1256.

4. Murthy M, Murthy B (2011) Amlodipine-induced petechial rash. J Postgrad Med 57: 341.

5. Orme S, da Costa D (1997) Drug points: Generalised pruritus associated with amlodipine. BMJ 315: 463.

6. Low L, Zaheri S, Wakelin S (2012) Amlodipine-induced linear IgA disease. Clin Exp Dermatol 37: 649-651.

7. Swale VJ, McGregor JM (2001) Amlodipine-associated lichen planus. Br J Dermatol 144: 920-921.

8. Patterson J (2016) Weedon's Skin Pathology. Cutaneous Drug Reactions 595-610.

9. Parlar B, Blazek C, Cazzaniga S (2014) Treatment of lower extremity telangiectasias in women by foam sclerotherapy vs. Nd: YAG laser: A prospective, comparative, randomized, openlabel trial. J Eur Acad Dermatol Venereol 29: 549-554.

10. Tepavcevic B, Matic P, Radak D (2012) Comparison of sclerotherapy, laser, and radiowave coagulation in treatment of lower extremity telangiectasias. J Cosmet Laser Ther 14: 239-242.

11. Munia MA, Wolosker N, Munia CG (2012) Comparison of laser versus Sclerotherapy in the treatment of lower extremity Telangiectases: A prospective study. Dermatol Surg 38: 635-639.

12. Peterson JD, Goldman MP, Weiss RA (2012) Treatment of Reticular and Telangiectatic leg veins: Double-blind, prospective comparative trial of Polidocanol and Hypertonic saline. Dermatol Surg 38: 1322-1330.

13. McCoy S, Evans A, Bmbs SN (1999) Sclerotherapy for leg Telangiectasia-A blinded comparative trial of Polidocanol and Hypertonic saline. Dermatol Surg 25: 381-386.

14. Kern P, Ramelet AA, Wutschert R (2004) Single-blind, Randomized study comparing Chromated glycerin, Polidocanol solution, and Polidocanol foam for treatment of Telangiectatic leg veins. Dermatol Surg 30: 367-372.

15. Cartee TV, Schleich M, Helm KF (2015) Granuloma Annulare arising in Tumescent Anesthesia injection sites after Endovenous ablation. Dermatol Surg 41: 1184-1186.

16. Goldman MP, Guex JJ, Weiss RM (2014) Sclerotherapy: Treatment of Varicose and Telangiectatic leg veins.

17. Korkomaz J, Maatouk I, Moutran R (2014) Nicolau livedoid dermatitis occurring after sclerotherapy. Vasc Med 19: 415-416.

18. Ramelet AA, Parmentier L (2010) Delayed Nicolau's Livedoid Dermatitis after ultrasound guided Sclerotherapy. Dermatol Surg 36: 155-158.

19. Shear N, Knowles S (2014) Treatment of Skin Disease. Drug Eruptions 197-199. 